\title{
Calculation of Equivalent and Toric Power in AddOn Lenses Based on a Monte Carlo Simulation
}

\author{
Achim Langenbucher $^{a}$ Jens Schrecker ${ }^{b}$ Alan Cayless ${ }^{c}$ Peter Hoffmann ${ }^{d}$ \\ Jascha Wendelstein ${ }^{\mathrm{e}}$ Nóra Szentmáryf,g \\ aDepartment of Experimental Ophthalmology, Saarland University, Homburg, Germany; ${ }^{b}$ Department of \\ Ophthalmology, Rudolf-Virchow-Clinics, Glauchau, Germany; 'School of Physical Sciences, The Open University, \\ Milton Keynes, UK; ${ }^{\mathrm{d} A u g e n-}$ und Laserklinik Castrop-Rauxel, Castrop-Rauxel, Germany; ${ }^{\text {DDepartment of }}$ \\ Ophthalmology, Johannes Kepler University Linz, Linz, Austria; fDr. Rolf M. Schwiete Center for Limbal \\ Stem Cell and Aniridia Research, Saarland University, Homburg, Germany; 9Department of Ophthalmology, \\ Semmelweis-University, Budapest, Hungary
}

\section{Keywords}

AddOn lens · Pseudophakic eye - Equivalent power · Toric power · Refraction · Monte Carlo simulation

\begin{abstract}
Introduction: Additional lenses implanted in the ciliary sulcus (AddOn) are one option for permanent correction of refractive error or generate pseudoaccommodation in the pseudophakic eye. The purpose of this paper was to model the power and magnification behaviour of toric AddOn and to show the effect sizes with a Monte Carlo simulation. Methods: Anonymized data of a cataractous population uploaded for formula constant optimization were extracted from the IOLCon platform. After filtering out data with refractive spherical equivalent (RSEQ) between -0.75 and $0.25 \mathrm{dpt}$ and refractive cylinder (RCYL) lower than 0.75 , for each of the $N$ $=6,588$ records, a toric AddOn was calculated which transfers the refraction error from spectacle plane to AddOn plane using a matrix-based calculation strategy based on lin-
\end{abstract}

Karger@karger.com www.karger.com/ore

Karger $\stackrel{\text { ' }}{5}$

GOPEN ACCESS
(C) 2022 The Author(s)

Published by S. Karger AG, Basel

This is an Open Access article licensed under the Creative Commons Attribution-NonCommercial-4.0 International License (CC BY-NC) (http://www.karger.com/Services/OpenAccessLicense), applicable to the online version of the article only. Usage and distribution for commercial purposes requires written permission. ear Gaussian optics. The equivalent (AddOnEQ) and toric (AddOnCYL) power of the AddOn and the overall lateral magnification change and meridional magnification were derived for the situations before and after AddOn implantation, and a linear modelling was fitted for all 4 parameters. Results: RSEQ is the dominant effect size in the prediction of AddOnEQ and overall change in magnification $(\Delta M)$, whereas the lens position (LP), corneal thickness (CCT), and mean corneal radius $\left(\mathrm{CP}_{\mathrm{a}}\right)$ play a minor role. In a simplified model, AddOnEQ can be estimated by $0.0179+1.4104$ RSEQ. RCYL and corneal radius difference $\left(\mathrm{CP}_{\mathrm{ad}}\right)$ are the dominant effect sizes in the prediction of AddOnCYL and the change in meridional magnification $\left(\triangle \mathrm{M}_{\text {mer }}\right)$, whereas $\mathrm{LP}, \mathrm{CCT}, \mathrm{CP}_{\mathrm{a}}$, and RSEQ play a minor role. In a simplified model, AddOnCYL can be predicted by $-0.0005+0.0328 \mathrm{CP}_{\mathrm{ad}}+1.4087 \mathrm{RCYL}$. Myopic eyes gain in overall magnification, whereas in hyperopic eyes, we observe a loss. Meridional distortion could be in general reduced to $35 \%$ on average with a toric AddOn. Conclusion: Our simulation shows that with a linear model, the equivalent and toric AddOn power, as well as overall change
Correspondence to:

Achim Langenbucher, achim.langenbucher@uks.eu 
in magnification, meridional distortion before and after AddOn implantation, and the reduction in meridional distortion, can be easily predicted from the biometric data in pseudophakic eyes with moderate refractive error.

(c) 2022 The Author(s).

Published by S. Karger AG, Basel

\section{Introduction}

With the development of optical biometry in cataract surgery and the launch of the IOLMaster as the first optical biometer in 1999, the reliability of biometric measures increased significantly. In contrast to the first biometer generation, modern biometers are capable of measuring all relevant axial distances such as central corneal thickness (CCT), phakic or pseudophakic aqueous depth, and crystalline or artificial lens thickness, as well as the vitreous depth with a high precision. In addition, lens power calculation concepts responded to these new measures and upgraded by the use of additional parameters, e.g., corneal or lens thickness to improve the prediction of an appropriate lens power. Some newest generation biometers are even capable of measuring the corneal topography instead of keratometry or the curvature of both corneal surfaces using Scheimpflug slit projection or optical coherence techniques.

But even with modern biometers and lens power calculation concepts, there are situations where finetuning after cataract surgery is required, and AddOn lenses are an appropriate solution [1-6]. In situations where the patient decides for the option of pseudophakic pseudoaccommodation only after cataract surgery, multifocal AddOn provide likewise a sufficient alternative [7-12]. Another indication for AddOn could be after TRIPLE or new TRIPLE procedure, where the cataractous lens was replaced during penetrating keratoplasty or DMEK/ DSAEK [3]. In those cases, the prediction of the appropriate lens power may fail due to the unpredictable or varying corneal power, and AddOn can be used to correct the persisting cylindric and equivalent refraction error [13]. In contrast to capsular bag lenses, where the risk during manipulation or exchange increases 2-3 months after cataract surgery, AddOn can be implanted, manipulated, exchanged, or explanted at any time after IOL surgery. But, the drawback of this reversibility is that AddOn may rotate even months or years after implantation, which could induce crossed cylinders and a deteriorated visual performance with toric models $[14,15]$.

In contrast to capsular bag intraocular lenses, the power of AddOn is not calculated on the basis of standard biometric measures [13]. As with phakic lenses, for the calculation, we require the refraction at spectacle plane which should be corrected, the axial distance between the spectacle back vertex and the corneal front vertex (vertex distance, VD), and the corneal curvature, as well as the axial position of the AddOn in the eye with respect to the corneal front vertex (lens position, LP) [16]. The cornea could either be considered as thin lens with one refracting surface or as thick lens with a corneal front surface and back surface curvature, each with equivalent power and astigmatism with axis at random. In contrast to capsular bag lenses which typically show a significant variation with respect to the axial position predicted in the lens calculation formula $[11,17,18]$, the variation in axial position of an AddOn shows only a minor variation, and we are able to predict the axial position of the AddOn from the measured pseudophakic anterior chamber depth and the axial distance between both IOL (typically between 0.3 and $0.5 \mathrm{~mm}$ ). Furthermore, as the refractive power of AddOn is typically in the lower range (in contrast to lenses in the capsular bag), the variation in axial position is of minor relevance [13].

In some clinical settings, the equivalent and toric power of an AddOn or phakic lens is derived from equivalent and cylindrical refraction using a fixed factor. This factor might be proper in many situations, but in some cases it may fail, and calculation of the AddOn power using a prediction model yields superior results [19-21]. The purpose of this paper is to evaluate the equivalent and toric power of AddOn lenses as well as the average and meridional change in object to image magnification by means of linear Gaussian optics using matrix algebra and to derive linear models with a Monte Carlo simulation to evaluate the effect of biometric measures such as refraction, corneal curvature, and axial length on AddOn position.

\section{Methods}

Dataset for the Monte Carlo Simulation

From a dataset of 21,340 data of a cataractous population which was uploaded for formula constant optimization to the IOLCon web-based platform (www.IOLCon.org), we extracted corneal front surface curvature data (flat and steep meridian including axis, mean radius $\left[\mathrm{CR}_{\mathrm{a}}\right]$, and radius difference $\left[\mathrm{CR}_{\mathrm{ad}}\right]$ ) as well as phakic anterior chamber depth data, axial length data, equivalent power of the capsular bag lens, and postoperative refraction data considered at spectacle plane (sphere, cylinder, and axis). The study was registered at the local ethics committee (Ethikkommission der Ärztekammer des Saarlandes, Reference No. 157/21). Informed consent of the patients was not required. 
Table 1. Descriptive data of the $A L$, pseudophakic $A C D, C C T$, mean radius of curvature $(C R)$ and radius difference between the flat and steep meridian $\left(C_{\mathrm{ad}}\right)$ of the corneal front surface, RSEQ, and RCYL of the pseudophakic eye before implantation of the AddOn lens at spectacle plane

\begin{tabular}{llllllll}
\hline & $\mathrm{AL}, \mathrm{mm}$ & $\mathrm{ACD}, \mathrm{mm}$ & $\mathrm{CCT}, \mu \mathrm{m}$ & $\mathrm{CR}_{\mathrm{a}}, \mathrm{mm}$ & $\mathrm{CR}_{\mathrm{ad}}, \mathrm{mm}$ & $\mathrm{RSEQ}, \mathrm{dpt}$ & $\mathrm{RCYL}, \mathrm{dpt}$ \\
\hline Mean & 23.83 & 4.90 & 542 & 7.78 & 0.28 & -0.26 & 1.49 \\
$\mathrm{SD}$ & 1.65 & 0.35 & 27 & 0.31 & 0.18 & 0.71 & 0.97 \\
Median & 23.59 & 4.90 & 541 & 7.78 & 0.24 & -0.25 & 1.25 \\
Minimum & 18.10 & 3.61 & 440 & 6.66 & 0.00 & -2.75 & 0.00 \\
Maximum & 33.04 & 6.34 & 651 & 8.89 & 1.50 & 2.12 & 0.50 \\
Cl lower bound & 21.39 & 4.33 & 498 & 7.27 & 0.06 & -1.5 & 0.25 \\
Cl upper bound & 26.68 & 5.49 & 586 & 8.29 & 0.61 & 0.87 & 3.25 \\
\hline
\end{tabular}

The data refer to arithmetic mean, standard deviation, median, minimum and maximum, and lower and upper bounds of the $95 \%$ confidence interval.

From axial length data, phakic anterior chamber depth, and equivalent power of the capsular bag lens, we made an estimation of the pseudophakic anterior chamber depth. The axial position of the AddOn was assumed to be on average $0.4 \pm 0.05 \mathrm{~mm}$ in front of the anterior vertex of the capsular bag lens (for the variation, a Gaussian distribution with a standard deviation [SD] of 0.05 was assumed).

Corneal front surface curvature was transferred to front surface power using corneal refractive index $\left(n_{\mathrm{C}}=1.376\right)$ and decomposed into equivalent power and astigmatic vector components in $\mathrm{C}_{\mathrm{a}} 0^{\circ}$ $\left(0^{\circ} / 90^{\circ}\right)$ and $\mathrm{C}_{\mathrm{a}} 45^{\circ}\left(45^{\circ} / 135^{\circ}\right)$ [22]. CCT was modelled by a normally distributed random sequence with a mean of $550 \mu \mathrm{m}$ and an $\mathrm{SD}$ of $20 \mu \mathrm{m}$. Mean corneal back surface curvature $\left(\mathrm{CR}_{\mathrm{p}}\right)$ was calculated from a front to back surface curvature ratio of $1.214 \pm 0.05$ according to the Liou-Brennan model eye and transferred to corneal back surface power using the refractive indices of cornea $n_{\mathrm{C}}$ and aqueous humour $n_{\mathrm{A}}$. From the astigmatic components $\mathrm{C}_{\mathrm{a}} 0^{\circ}$ and $\mathrm{C}_{\mathrm{a}} 45^{\circ}$, the respective vector components for the corneal back surface $\left(\mathrm{C}_{\mathrm{p}} 0^{\circ}\right.$ and $\left.\mathrm{C}_{\mathrm{p}} 45^{\circ}\right)$ were extracted using a ratio of $\left(n_{\mathrm{A}}-n_{\mathrm{C}}\right) /$ $\left(n_{\mathrm{C}}-1\right) \pm 0.005$. Spherical equivalent of refraction (RSEQ), refractive cylinder (RCYL), and cylinder axis (RA) could be directly extracted from the postcataract refraction data.

From the entire dataset, we filtered the data used for our lens power calculation and Monte Carlo simulation as follows:

- Data where toric capsular bag lenses were implanted were excluded;

- Data where the RSEQ was within limits $(-0.75 ; 0.25) \mathrm{dpt}$ and the RCYL was within limits of $(0 ; 1.0)$ dpt were not considered, as in these situations, implantation of an AddOn is contraindicated.

Finally, $N=6,588$ data could be used for our Monte Carlo simulation.

\section{Calculation of the AddOn}

For each data, we performed calculation of a toric AddOn lens based on paraxial Gaussian optics using $4 \times 4$ matrix algebra [13, $18,22]$. For all refracting surfaces (pseudophakic spectacle correction, corneal front surface, and corneal back surface), we derived $4 \times 4$ refraction matrices, and for all homogeneous optical interspaces (VD, CCT, and LP), we defined $4 \times 4$ translation matrices. From all the refraction and translation matrices, a $4 \times 4$ system matrix was defined. Assuming the aperture stop of the eye being located at the AddOn plane LP behind the cornea, we defined the chief ray passing through the centre of the aperture [23]. This selection of the chief ray is necessary as the optical system described by the $4 \times 4$ system matrix is uncorrected. In a next step, an optical system was set up with the situation after implanting the toric AddOn. This system was assumed to transfer the refraction error at spectacle plane (before implantation of the AddOn) fully into the eye to the LP plane. Again, the $4 \times 4$ system matrix and the chief ray was determined for the postoperative situation, and from the system matrices of the preoperative and the postoperative situation, we extracted the equivalent power (AddOnEQ) and the cylindrical power (AddOnCYL) as well as the implantation axis of the toric AddOn lens using an eigenvalue decomposition [23]. From the chief ray angle for the situation before and after implantation of the AddOn, we calculated the meridional magnification disparity of the optical system for both situations as well as the relative object to image magnification change using eigenvalue decomposition. What we get out is the meridional distortion before and after implantation of the toric AddOn as well as the change in overall magnification due to transferring the refraction from spectacle plane to the AddOn plane LP.

\section{Evaluation of the Data}

The AddOn equivalent power and toric power, the relative overall change in magnification due to implantation of the AddOn ( $\triangle \mathrm{M}$ in \%), and the meridional distortion of the pseudophakic eye before $\left(M_{\text {mer }}\right.$ pre in \%) and after $\left(M_{\text {mer }}\right.$ post in \%) implantation of the toric AddOn, as well as the ratio of meridional distortion before to after AddOn implantation $\left(\Delta \mathrm{M}_{\mathrm{mer}}\right.$ in $\left.\%\right)$, were documented for all $N=6,588$ data. Then, multivariable linear models were set up to describe the equivalent power of the AddOn (AddOnEQ) as a function of LP, CCT, CR , and RSEQ and to describe the toric power of the AddOn (AddOnCYL) as a function of LP, CCT, $\mathrm{CR}_{\mathrm{a}}, \mathrm{CR}_{\mathrm{ad}}$, RSEQ, and RCYL, respectively. In addition, multivariable linear models were derived to predict the relative change in overall magnification as a function of LP, CCT, $\mathrm{CR}_{\mathrm{a}}$, and RSEQ and to describe the relative change in meridional magnification as a function of LP, CCT, $\mathrm{CR}_{\mathrm{a}}, \mathrm{CR}_{\mathrm{ad}}$, RSEQ, and RCYL due to implantation of a toric AddOn lens which transfers the refraction error from spectacle plane to LP. All biometric data and 


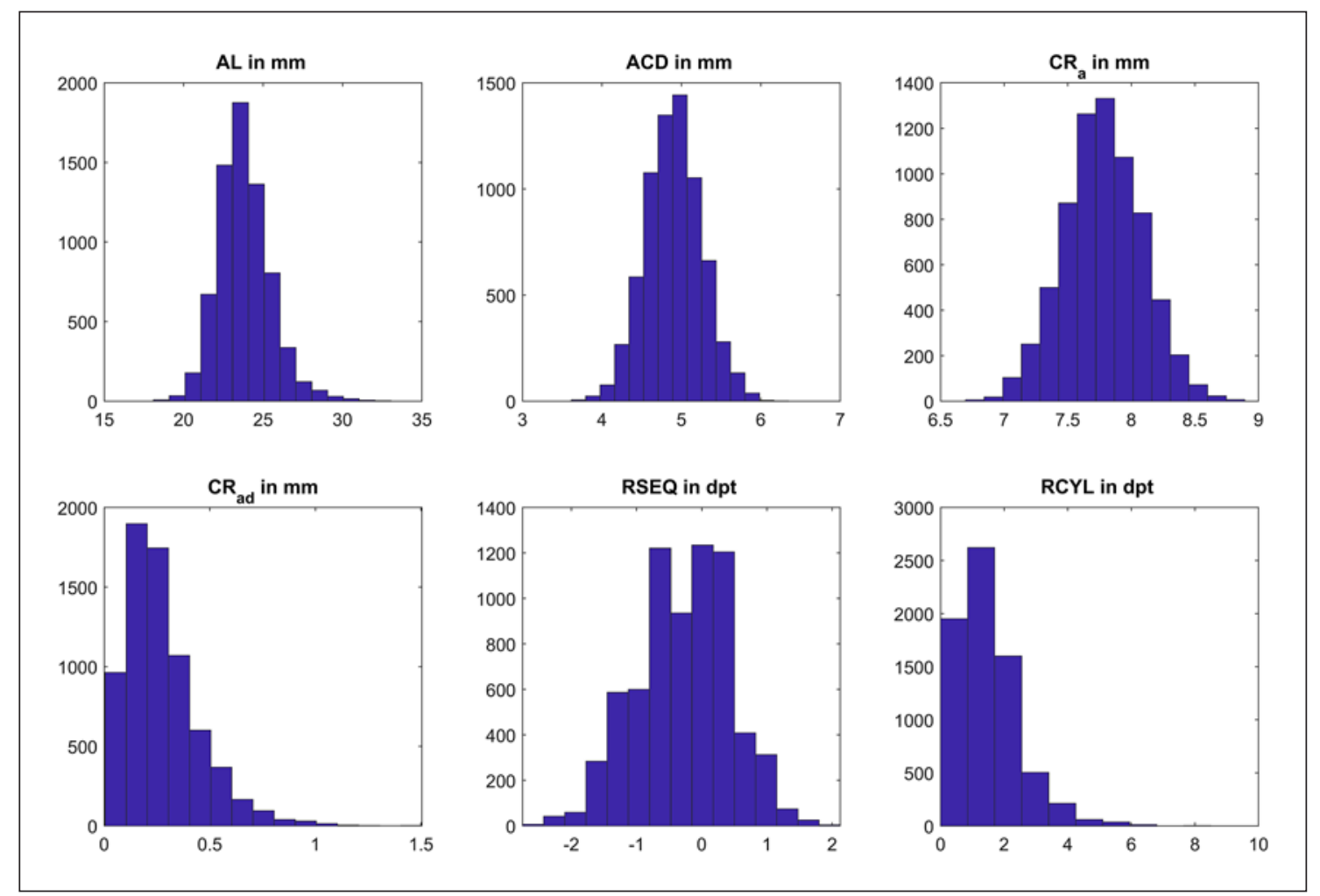

Fig. 1. Histograms of the distribution of $A L$, pseudophakic $A C D, C R_{a}, C_{a d}$, and SEQ and RCYL of spectacle refraction. AL, axial length; ACD, anterior chamber depth.

power data of the AddOn, as well as magnification data of our dataset, were described exploratively using mean, standard deviation, median, and minimum and maximum value, as well as $95 \%$ confidence interval.

\section{Results}

Table 1 shows a listing of the mean value, standard deviation, median, and minimum and maximum, as well as the $95 \%$ confidence intervals, of all biometric parameters used for this Monte Carlo simulation for the filtered dataset $(N=6,588$ cases). The axial length is not used for calculation of the AddOn, but is required to derive the size of the retinal image. The respective histograms of the distributions of parameters except the CCT are displayed in Figure 1.

Figure 2 shows the scatterplot with the correlation of the axis of RCYL and corneal astigmatism together with the fitted kernel distributions separated for low (up to 0.5 $\mathrm{dpt})$, moderate (0.5-1.0 dpt), high (1.0-2.0 dpt), and excessive values (more than $2.0 \mathrm{dpt}$ ) of RCYL. Cylinder axes around $0^{\circ}$ (with the rule) or around $90^{\circ}$ (against the rule) are much more prevalent than cylinder values in oblique axes (around $45^{\circ}$ or $135^{\circ}$ ). In the subgroup with low RCYL values, the axis of RCYL and the axis of corneal astigmatism show much more scatter compared to the groups with moderate, high, or excessive RCYL values.

Table 2 lists the results of the AddOn power calculation as well as the calculation of magnification due to implantation of an AddOn in terms of mean, standard deviation, median, range, and confidence interval. A comparison of the AddOnEQ with RSEQ and AddOnCYL with RCYL explains that the average and cylindrical power correction at AddOn plane is systematically higher compared to the correction with spectacles before AddOn implantation. For our dataset, the RSEQ before AddOn implantation was on average slightly myopic, and therefore patients on average benefit in magnification with AddOn implantation. Due to AddOn implantation, the meridional image distortion could be significantly reduced on average from $1.81 \%$ to $0.68 \%$, and the ratio between meridional distortions before to after AddOn implantation was on average 2.86. Figure 3 displays the ratio 


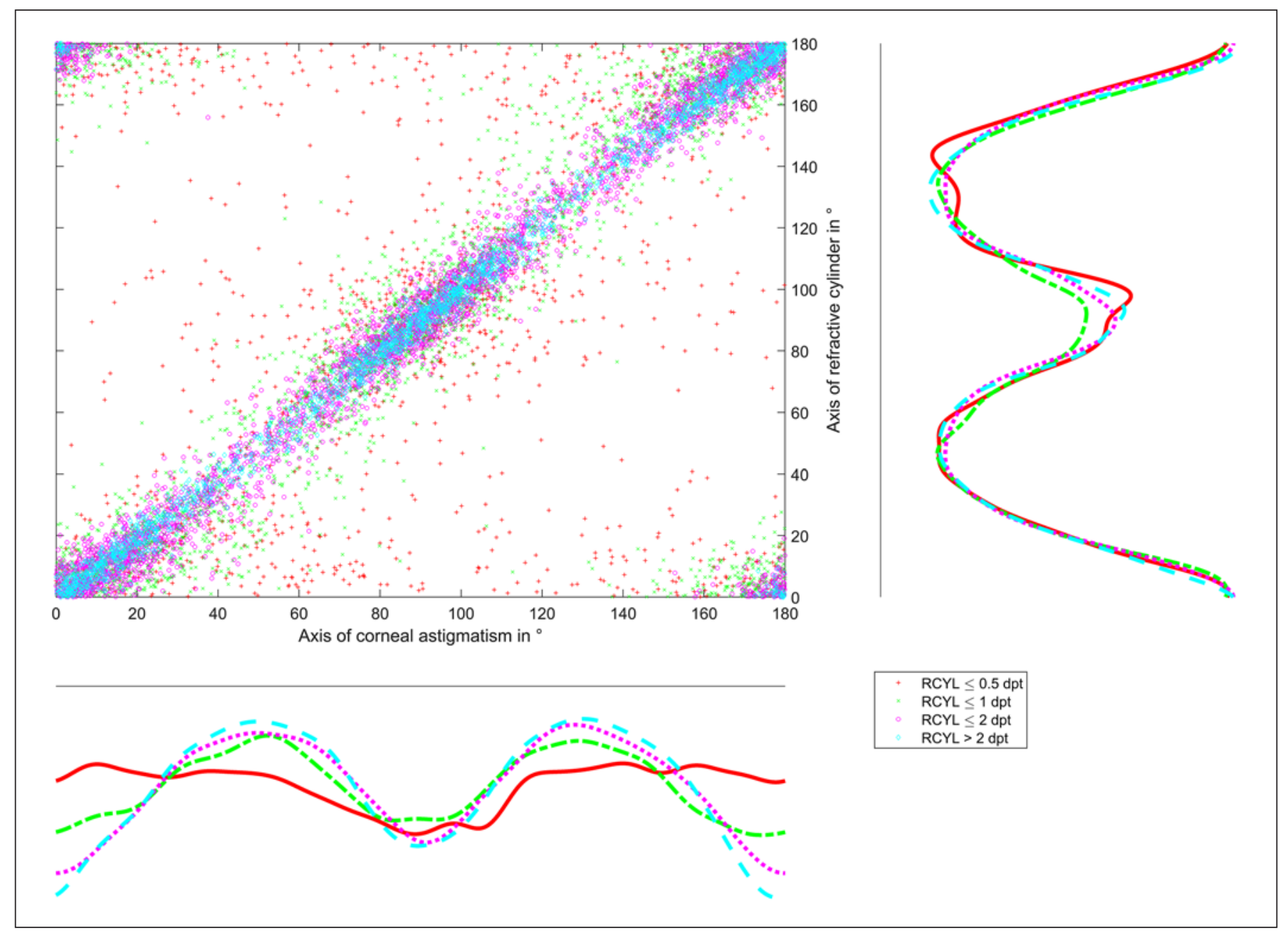

Fig. 2. Scatterplot of the axis of RCYL and the axis of corneal astigmatism grouped for RCYL. For higher values of RCYL, the axis of RCYL and the axis of corneal astigmatism coincide much better than for lower values of RCYL. The kernel distributions in the plot show that RCYL and corneal astigmatism show the highest prevalence with axes around $0 / 180^{\circ}$ (with the rule), followed by axes around $90^{\circ}$ (against the rule).

Table 2. Descriptive data of the equivalent power (AddOnEQ) and toric power (AddOnCYL) of the AddOn lens, overall change in magnification due to implantation of the AddOn lens $(\Delta M)$, meridional distortion in the spectacle corrected pseudophakic eye before $\left(M_{\text {mer }}\right.$ pre) and after implantation of the AddOn lens $\left(M_{\text {mer }}\right.$ post $)$, and the ratio of meridional distortion before to after implantation of the AddOn lens $\left(\Delta \mathrm{M}_{\text {mer }}\right)$

\begin{tabular}{lllllll}
\hline$N=6,588$ & AddOnEQ, dpt & AddOnCYL, dpt & $\Delta \mathrm{M}, \%$ & $\mathrm{M}_{\text {mer }}$ pre, \% & $\mathrm{M}_{\text {mer }}$ post, \% & $\Delta \mathrm{M}_{\text {mer, }} \%$ \\
\hline Mean & -0.35 & 2.11 & 0.44 & 1.81 & 0.69 & 2.85 \\
SD & 1.99 & 1.38 & 1.17 & 1.17 & 0.45 & 1.39 \\
Median & -0.34 & 1.78 & 0.41 & 1.55 & 0.59 & 2.63 \\
Minimum & -3.67 & 0.00 & -3.58 & 0.01 & 0.01 & 0.11 \\
Maximum & 3.31 & 11.65 & 4.47 & 10.37 & 3.91 & 9.97 \\
Cl lower bound & -2.04 & 0.36 & -1.44 & 0.37 & 0.15 & 1.10 \\
Cl upper bound & 1.26 & 4.73 & 2.43 & 4.03 & 1.55 & 5.49 \\
\hline
\end{tabular}

The data refer to arithmetic mean, standard deviation, median, minimum and maximum, and lower and upper bounds of the $95 \%$ confidence interval. 


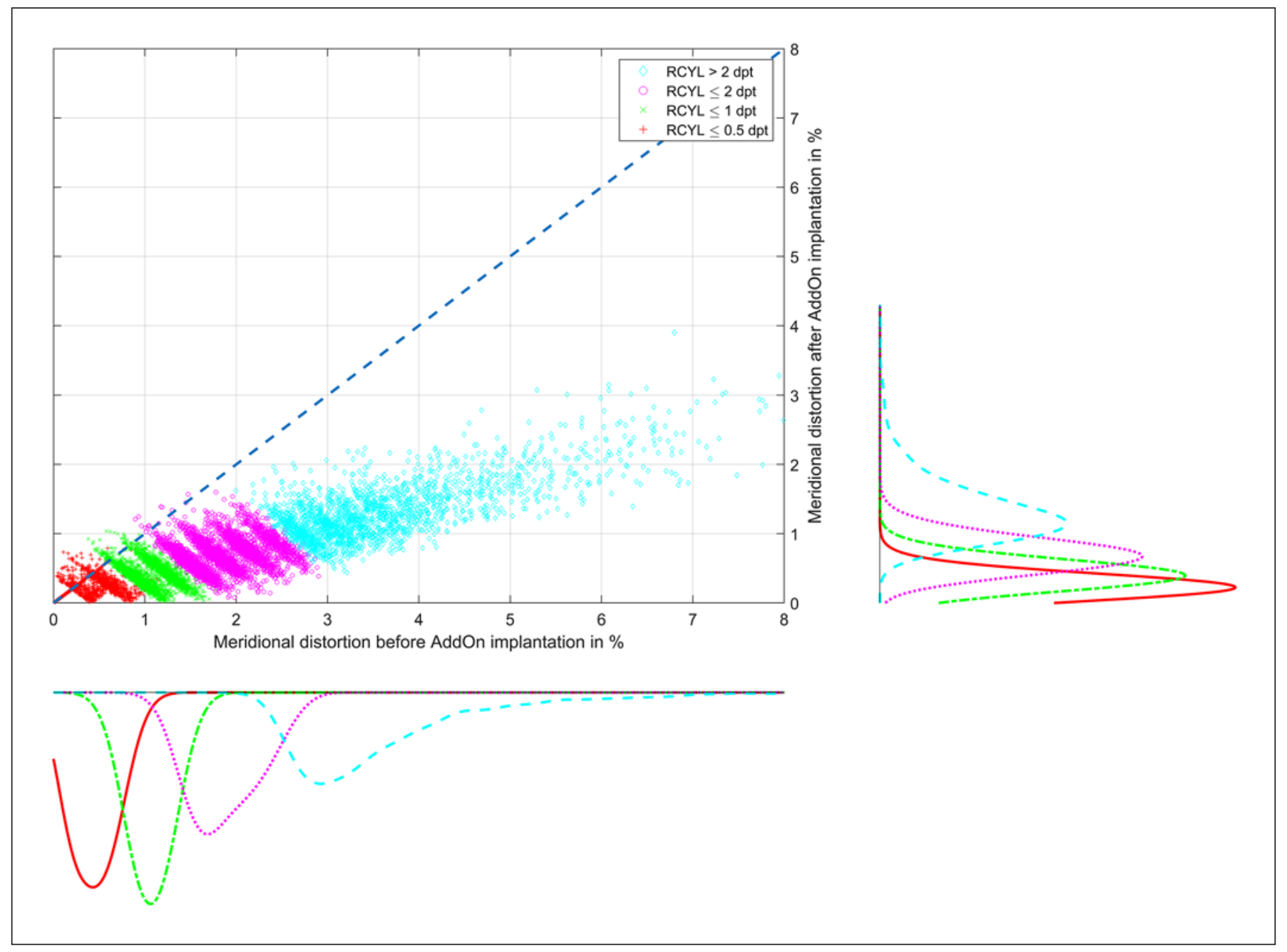

Fig. 3. Meridional distortion due to ocular astigmatism with spectacle correction ( $x$ axis, before implantation of an AddOn lens) and ocular astigmatism corrected with an AddOn lens ( $y$ axis, after implantation of an AddOn lens). The image distortion is systematically lower with AddOn lenses, especially with high amounts of pre-existing meridional distortions (with large astigmatism). The blue line indicates that meridional distortion does not change due to implantation of an AddOn lens.

of meridional image distortion $M_{\text {mer }}$ pre to $M_{\text {mer }}$ post separated low, moderate, high, and excessive RCYL. The benefit towards a meridionally eikonic image (without meridional distortion) is much higher with a pre-existing high or excessive RCYL compared to a pre-existing low RCYL.

\section{Model 1}

The multivariable linear prediction model for the equivalent power of the AddOn (AddOnEQ) as a function of LP, CCT, $\mathrm{CR}_{a}$, and RSEQ is defined by AddOnEQ $=-0.0050-0.0269 \mathrm{LP}-0.0272 \mathrm{CCT}+0.0204$ $\mathrm{CR}_{\mathrm{a}}+1.4103$ RSEQ.
The root mean squared error of the fit: 0.0365 and $R^{2}=0.999$. The $F$ value/significance level of this fit compared to a constant model was 1.79 e $06 / p<0.0001$.

\section{Model 2}

The multivariable linear prediction model for the overall change in magnification due to the AddOn implantation $(\triangle \mathrm{M})$ as a function of LP, CCT, $\mathrm{CR}_{\mathrm{a}}$, and RSEQ is defined by $\Delta \mathrm{M}=-0.0968+0.0280 \mathrm{LP}+0.0180$ CCT $-0.0048 \mathrm{CR}_{\mathrm{a}}-1.6509$ RSEQ.

The root mean squared error of the fit: 0.0279 and $R^{2}=0.998$. The $F$ value/significance level of this fit compared to a constant model was 4.20 e $06 / p<0.0001$. 
Fig. 4. Scatterplots with linear regressions which explain the isolated effects of the parameters used in models 1 and 2 for prediction of equivalent power of the AddOn lens (AddOnEQ) and overall change on magnification due to implantation of an AddOn lens $(\Delta \mathrm{M})$. For both models, the spherical equivalent outperforms the effect of axial LP, CCT, and mean $\mathrm{CR}_{\mathrm{a}}$. The fit lines refer to the linear fit in terms of minimizing the root mean squared fit error.

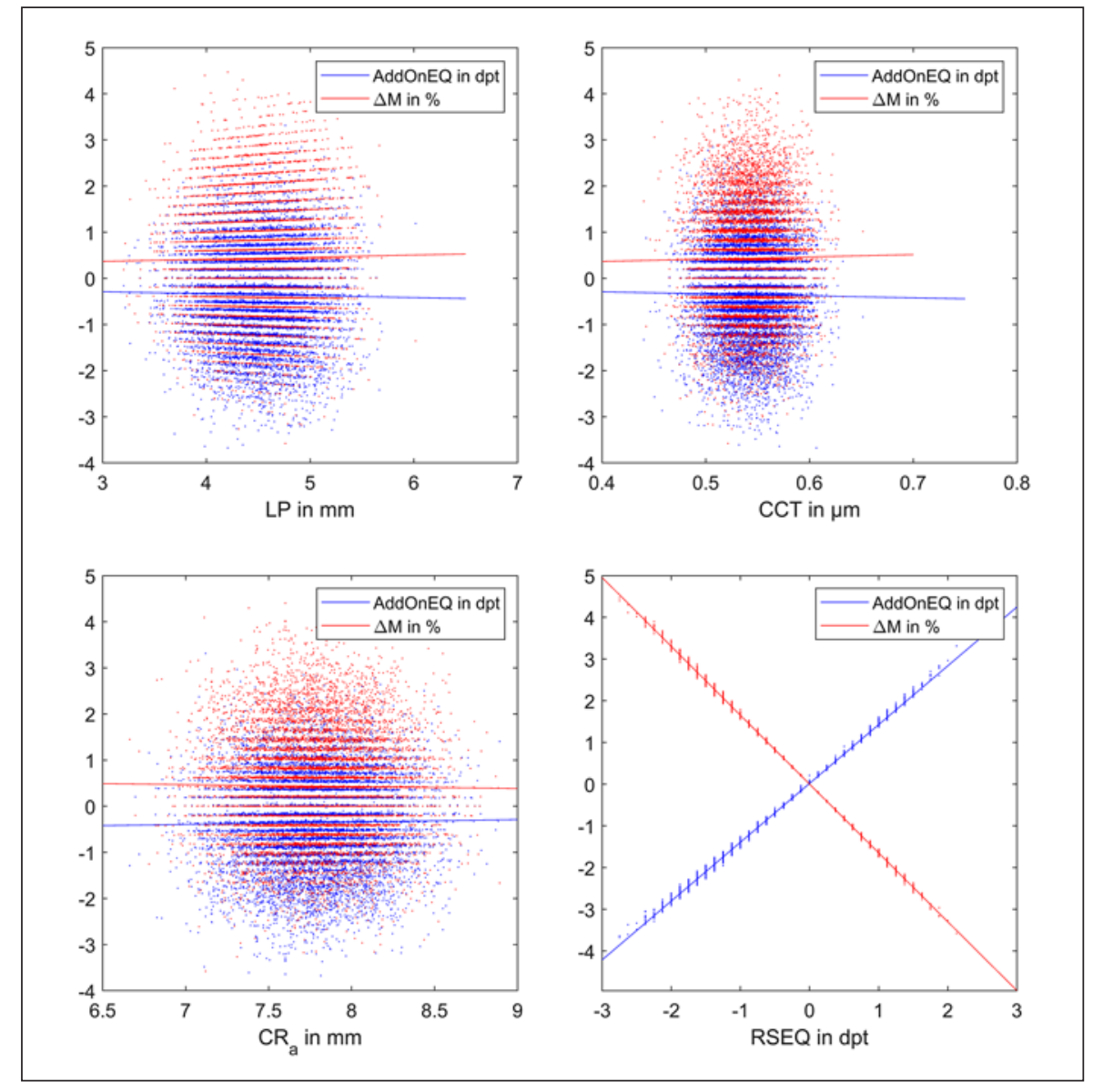

\section{Model 3}

The multivariable linear prediction model for the toric power of the AddOn (AddOnCYL) as a function of LP, CCT, $\mathrm{CR}_{\mathrm{a}}, \mathrm{CR}_{\mathrm{ad}}$, RSEQ, and RCYL is defined by AddOn$\mathrm{CYL}=0.0312+0.1601 \mathrm{LP}+0.1643 \mathrm{CCT}-0.1071 \mathrm{CR}_{\mathrm{a}}+$ $0.0198 \mathrm{CR}_{\mathrm{ad}}+0.0445 \mathrm{RSEQ}+1.4135 \mathrm{RCYL}$.

The root mean squared error of the fit: 0.0494 and $R^{2}=0.995$. The $F$ value/significance level of this fit compared to a constant model was 1.24 e $06 / p<0.0001$.

\section{Model 4}

The multivariable linear prediction model for the ratio of meridional distortion before to after AddOn implantation $\left(\Delta \mathrm{M}_{\mathrm{mer}}\right.$ in \%) as a function of LP, CCT, $\mathrm{CR}_{\mathrm{a}}, \mathrm{CR}_{\mathrm{ad}}$, RSEQ, and RCYL is defined by $\Delta \mathrm{M}_{\text {mer }}=-0.6654+0.1600$ $\mathrm{LP}+0.14198 \mathrm{CCT}-0.0170 \mathrm{CR}_{\mathrm{a}}+0.0080 \cdot \mathrm{CR}_{\mathrm{ad}}-0.0027$ RSEQ + 1.6490 RCYL.

The root mean squared error of the fit: 0.0379 and $R^{2}=0.991$. The $F$ value/significance level of this fit compared to a constant model was 2.87 e $06 / p<0.0001$.
The isolated effects of LP, CCT, CR AddOnEQ and $\triangle \mathrm{M}$ are displayed for a better visualization in scatterplots in Figure 4 together with the fit lines in terms of a minimization of the least squares fit error. Even if LP, CCT, and $\mathrm{CR}_{\mathrm{a}}$ show a statistically significant effect, RSEQ dominates the multivariate models 1 and 2, and therefore a simplification to AddOnEQ $=0.0179+$ 1.4104 RSEQ (root mean squared fit error: 0.0383 ) and to $\Delta \mathrm{M}=0.0016-1.651$ RSEQ (root mean squared fit error: 0.0297 ) seems to be appropriate.

The isolated effects of LP, CCT, $\mathrm{CR}_{\mathrm{a}}, \mathrm{CR}_{\mathrm{ad}}$, RSEQ, and RCYL on AddOnCYL and $\triangle \mathrm{M}_{\text {mer }}$ are displayed for a better visualization in scatterplots in Figure 5 together with the fit lines in terms of a minimization of the least squares fit error. Even if LP, CCT, $\mathrm{CR}_{\mathrm{a}}$, and RSEQ show a statistically significant effect, RCYL and $\mathrm{CR}_{\mathrm{ad}}$ are the major effect sizes in the multivariate models 3 and 4 , and therefore a simplification to AddOnCYL $=-0.0005+0.0328 \mathrm{RCYL}$ $+1.4087 \mathrm{CR}_{\mathrm{ad}}$ (root mean squared fit error: 0.0887 ) and to $\Delta \mathrm{M}_{\text {mer }}=0.0013+0.0083 \mathrm{RCYL}+1.6482 \mathrm{CR}_{\mathrm{ad}}$ seems to be a sufficient fit (root mean squared fit error: 0.0699). 


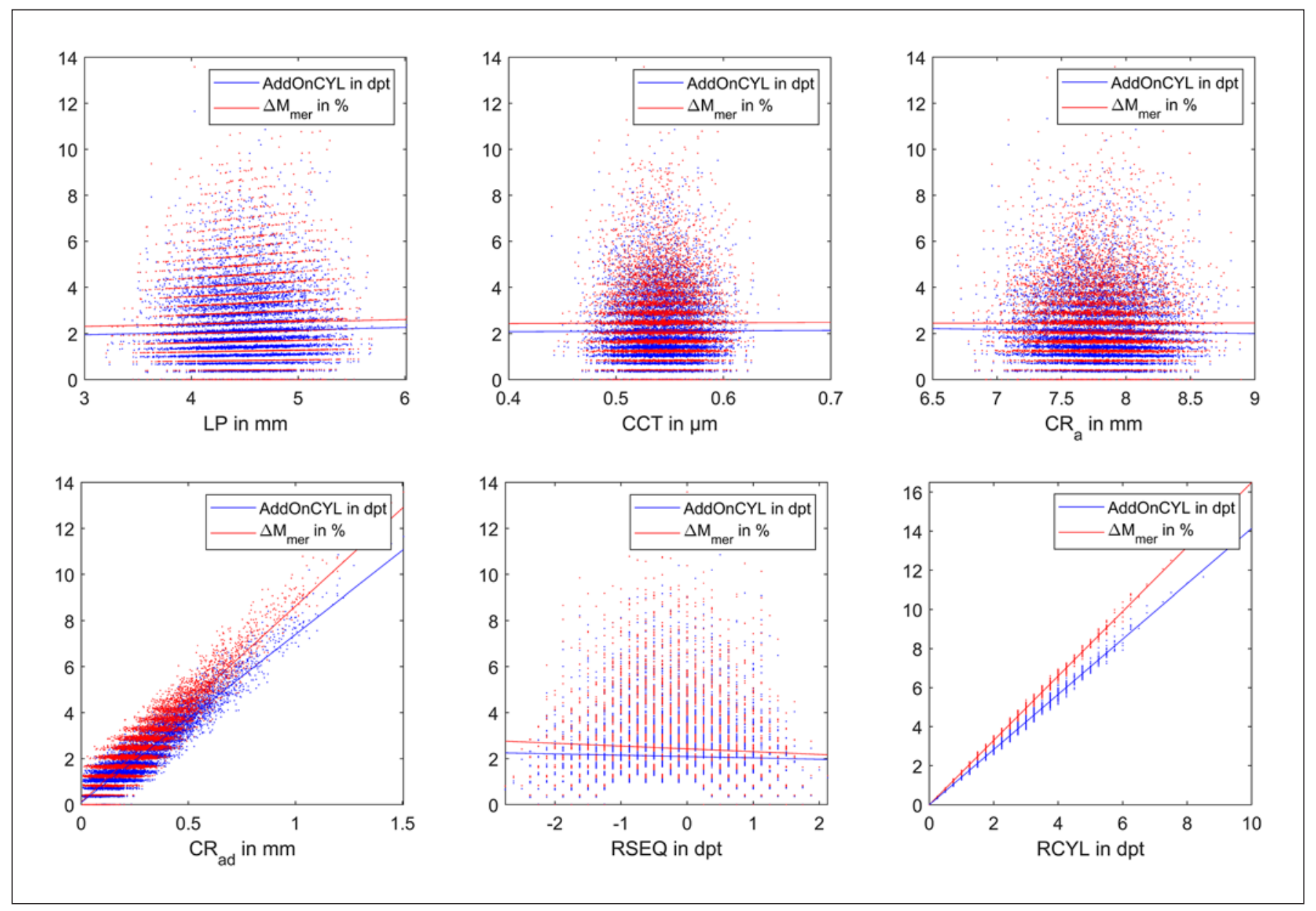

Fig. 5. Scatterplots with linear regressions which explain the isolated effects of the parameters used in models 3 and 4 for prediction of toric power of the AddOn lens (AddOnCYL) and the lateral distortion ratio before to after implantation of an AddOn lens $\left(\Delta \mathrm{M}_{\mathrm{mer}}\right)$. For both models, the RCYL and the difference between corneal radii in both cardinal meridians $\left(\mathrm{CR}_{\mathrm{ad}}\right)$ outperform the effect of axial LP, CCT, mean $\mathrm{CR}_{\mathrm{a}}$, and SEQ. The fit lines refer to the linear fit in terms of minimizing the root mean squared fit error.

\section{Discussion}

AddOn lenses are a convenient and effective option to correct refractive deviations in pseudophakic eyes. An additional benefit of these lenses is their positioning in the sulcus ciliaris which allows for an exchange or explantation at any time, and the surgical procedure of implanting or removing this IOL is relatively simple without endangering surrounding structures. In many cases, the present refractive error is within a moderate range, and a fast and easy method to predict the necessary power for a correcting AddOn would be useful. For this purpose, we derived linear models by means of linear Gaussian optics with a Monte Carlo simulation.

Monte Carlo Simulation for Toric AddOn Lenses
Monte Carlo simulations are statistical methods used to analyse the effect of dependent variables on target variables and to define (simplified) models for a prediction based on a large number of cases. As a starting point for our study served a large dataset of real clinical data. These data were first used to predict missing parameters such as the pseudophakic anterior chamber depth for estimation of the axial LP. To resemble realistic conditions, we filtered out data where the spherical equivalent was within -0.75 and $0.25 \mathrm{dpt}$ and RCYL within 0 and $0.75 \mathrm{dpt}$. Next, data were used to derive the power of an AddOn which corrects the eye for emmetropia, which means that spectacle correction of the pseudophakic eye is transferred to the AddOn plane. 
Calculations were performed with linear Gaussian optics restricted to the paraxial space using $4 \times 4$ matrix algebra $[17,22]$. The principle of the calculation scheme used for this Monte Carlo simulation has been already presented in previous papers [13]. The respective system matrices for the situation before and after AddOn implantation provide beside the equivalent and toric power (and axis) of the AddOn also lateral magnification in both cardinal meridians derived with an eigenvalue decomposition. As the optical system of the anterior eye segment from spectacle to the AddOn plane is uncorrected, the rays in the ray bundle will not converge properly, and therefore a selection of a unique ray (in our situation the chief ray) is necessary to get out lateral magnification. This chief ray should pass through the centre of the aperture which was assumed to be located at the AddOn plane. Besides matrix calculation, there are several options for calculation such as vergence transform or raytracing strategies without restrictions to paraxial optics if all refractive surfaces are fully described [13, 17, 22].

The characteristics of the dataset used for Monte Carlo simulation are described exploratively in Table 1 and in Figure 1. In Figure 2, we read directly that for higher values of RCYL, the coupling of the axis of RCYL and the axis of corneal astigmatism is very high, but for lower values, especially for RCYL up to $0.5 \mathrm{dpt}$, we see a large scatter. This scatter is mostly likely a result of measurement uncertainties of subjective refraction and corneal shape. It is obvious that due to transformation of the refractive correction from spectacle plane to AddOn plane, the spectacle minification is reduced for myopes, and the spectacle magnification is reduced for hyperopes as shown with the parameter $\Delta \mathrm{M}$. But as a consequence, we should also notice that meridional distortion as the difference of lateral magnification in both cardinal meridians subdivided by the average magnification is systematically reduced if a spectacle corrected cylinder is transferred to AddOn plane. From Figure 3, we see that more or less in all cases, meridional distortion could be reduced systematically especially with higher values of RCYL. On average, the meridional distortion was reduced to $1 / 2.85=35 \%$ as shown in Table 2 (column with $\left.\Delta \mathrm{M}_{\text {mer }}\right)$.

As we can read out from the scatterplots in Figure 4, the axial LP, CCT, and corneal front surface radius $\left(\mathrm{CR}_{\mathrm{a}}\right)$ do not play a major role for the prediction of the equivalent power of the AddOn and the change in overall magnification due to implantation of an AddOn. The spherical equivalent of refraction is the dominating effect size for prediction of AddOnEQ and $\Delta \mathrm{M}$, and therefore a simplified prediction model for the AddOn equivalent power with AddOnEQ $=0.0179+1.4106$ RSEQ (with intercept) or a simple scaling with AddOnEQ $=1.4022$ RSEQ (without intercept) seems to be rectified.

Figure 5 shows that the axial LP, CCT, and the mean corneal front surface radius, as well as spherical equivalent of refraction, do not play a major role in prediction of toric power of the AddOn or the change in meridional distortion. The dominating effect sizes are the difference of both corneal front surface radii $\left(\mathrm{CR}_{\mathrm{ad}}\right)$ and the RCYL. Therefore, simplified prediction models for AddOnCYL $=-0.0005+0.0328 \mathrm{CR}_{\mathrm{ad}}+1.4087 \mathrm{RCYL}$ and $\Delta \mathrm{M}_{\text {mer }}=0.0013+0.0083 \mathrm{CR}_{\mathrm{ad}}+1.6482 \mathrm{RCYL}$ which include $\mathrm{CR}_{\mathrm{ad}}$ and RCYL only seem to be sufficient.

In general, in contrast to calculation strategies of capsular bag lenses, there are only rare papers on calculation of AddOn lenses or phakic lenses [13, 19, 20]. In many situations, clinicians use simple scaling factors for determination of AddOnEQ or AddOnCYL powers around 1.4 , and the present study shows that for the situation of AddOn, such a simplification performs quite well in clinical routine. But calculations with such simple scaling factor do not consider the individual characteristics of a patient and give no insight what happens if a toric AddOn shows some deviation from the target axis. A calculation scheme as it is used here considers the preoperative refraction (RSEQ, RCYL, and axis) and corneal shape $\left(\mathrm{CRa}, \mathrm{CR}_{\mathrm{ad}}\right.$, and axis), as well as vertex distance (which was assumed to be $12 \mathrm{~mm}$ in this study) and the predicted LP derived from the pseudophakic anterior chamber depth and the vault of the lens. As this calculation scheme deals with crossed cylinders, axes of RCYL and corneal astigmatism could be at random. And in addition, the prediction of overall magnification gain or loss due to AddOn implantation as well as the prediction of change (decrease) in meridional image distortion due to toric AddOn implantation might be a useful tool for patient counselling or indication of AddOn.

In conclusion, this Monte Carlo simulation showed that prediction of equivalent power and toric power of an AddOn could be properly done with simple (multivariable) linear models. In addition, these models allow for a prediction of change in overall image magnification and change in meridional distortion which may assist in patient counselling and decision-making for AddOn lens implantation. 


\section{Statement of Ethics}

This study was registered at the local Ethics Review Board (Ethikkommission der Ärztekammer des Saarlandes, registration 157/21). An ethical approval or a written informed consent from participants was not necessary.

\section{Conflict of Interest Statement}

The authors have no conflicts of interest to declare.

\section{Funding Sources}

Not applicable.

\section{Author Contributions}

Achim Langenbucher: planning of the study, calculations and programming, and scientific writing. Jens Schrecker: clinical assistance and critical revision. Alan Cayless: data interpretation, scientific writing, and critical revision. Peter Hoffmann: clinical assistance, critical revision, and data interpretation. Jascha Wendelstein: clinical assistance, critical revision, and data interpretation. Nóra Szentmáry: planning of the study, clinical assistance, and critical revision.

\section{Data Availability Statement}

The anonymized raw data that were analysed in the study could be provided on request.

\section{References}

1 Basarir B, Kaya V, Altan C, Karakus S, Pinarci EY, Demirok A. The use of a supplemental sulcus fixated IOL (humanoptics Add-On IOL) to correct pseudophakic refractive errors. Eur J Ophthalmol. 2012;22(6):898-903.

2 Gundersen KG, Potvin R. Refractive and visual outcomes after implantation of a secondary toric sulcus intraocular lenses. Clin Ophthalmol. 2020;1814:1337-42.

3 Hassenstein A, Niemeck F, Giannakakis K, Klemm M. Toric add-on intraocular lenses for correction of high astigmatism after pseudophakic keratoplasty. Ophthalmologe. 2017; 114(6):549-55.

4 Hengerer FH, Conrad-Hengerer I. Pseudophacic additive lenses. Klin Monbl Augenheilkd. 2017;234(12):e43-55.

5 Sauder G. Secondary toric intraocular lens implantation in pseudophakic eyes. The addon IOL system. Ophthalmologe. 2007; 104(12):1041-5.

6 Thomas BC, Auffarth GU, Reiter J, Holzer MP, Rabsilber TM. Implantation of threepiece silicone toric additive IOLs in challenging clinical cases with high astigmatism. J Refract Surg. 2013;29(3):187-93.

7 Albayrak S, Comba ÖB, Karakaya M. Visual performance and patient satisfaction following the implantation of a novel trifocal supplementary intraocular lens. Eur J Ophthalmol. 2020;2020:1120672120969042.

8 Gekeler K, Gekeler F. Multifocal and add-on intraocular lenses. Klin Monbl Augenheilkd. 2014;231(10):10371039-50.
9 Gerten G, Kermani O, Schmiedt K, Farvili E, Foerster A, Oberheide U. Dual intraocular lens implantation: monofocal lens in the bag and additional diffractive multifocal lens in the sulcus. J Cataract Refract Surg. 2009; 35(12):2136-43.

10 Palomino-Bautista C, Sánchez-Jean R, Carmona Gonzalez D, Romero Domínguez M, Castillo Gómez A. Spectacle independence for pseudophakic patients: experience with a trifocal supplementary add-on intraocular lens. Clin Ophthalmol. 2020;14:1043-54.

11 Schrecker J, Kroeber S, Eppig T, Langenbucher A. Additional multifocal sulcus-based intraocular lens: alternative to multifocal intraocular lens in the capsular bag. J Cataract Refract Surg. 2013;39(4):548-55

12 Schrecker J, Langenbucher A. Visual performance in the long term with secondary addon versus primary capsular bag multifocal intraocular lenses. J Refract Surg. 2016;32(11): 742-7.

13 Langenbucher A, Viestenz A, Szentmáry N, Seitz B, Viestenz A. Calculation of pseudophakic and phakic toric lenses for correction of corneal astigmatism-theory and clinical aspects. Klin Monbl Augenheilkd. 2008;225(6): 541-7.

14 Boutillier G, Gueudry J, Aouidid S, Muraine M. Sudden rotation and technique for repositioning Add-On ${ }^{\circledast}$ piggy-back sulcus toric intraocular lenses. J Fr Ophtalmol. 2021;12(21): S018100033-55124.

15 Muñoz G, Albarrán-Diego C, Belda L, Rohrweck S. Add-on sulcus-based versus primary in-the-bag multifocal intraocular lens: intraindividual study. J Refract Surg. 2014; 30(5):320-5.
16 Langenbucher A, Szentmáry N, Seitz B. Calculating the power of toric phakic intraocular lenses. Ophthalmic Physiol Opt. 2007;27(4): 373-80.

17 Langenbucher A, Reese S, Sauer T, Seitz B. Matrix-based calculation scheme for toric intraocular lenses. Ophthalmic Physiol Opt. 2004;24(6):511-9.

18 Langenbucher A, Seitz B. Computerized calculation scheme for bitoric eikonic intraocular lenses. Ophthalmic Physiol Opt. 2003; 23(3):213-20.

19 Eppig T, Gillner M, Walter S, Viestenz A, Langenbucher A. Calculation of phakic intraocular lenses. Klin Monbl Augenheilkd. 2011; 228(8):690-7.

20 Eppig T, Viestenz A, Seitz B, Langenbucher A. Calculation of pseudophakic toric intraocular lenses. Klin Monbl Augenheilkd. 2011;228(8): 681-9.

21 Holladay JT. Refractive power calculations for intraocular lenses in the phakic eye. Am J Ophthalmol. 1993;116(1):63-6.

22 Langenbucher A, Viestenz A, Szentmáry N, Behrens-Baumann W, Viestenz A. Toric intraocular lenses-theory, matrix calculations, and clinical practice. J Refract Surg. 2009; 25(7):611-22.

23 Langenbucher A, Viestenz A, Seitz B, Brünner $\mathrm{H}$. Computerized calculation scheme for retinal image size after implantation of toric intraocular lenses. Acta Ophthalmol Scand. 2007;85(1):92-8. 